\title{
Fictitious flux confinement: magnetic pairing in coupled spin chains or planes
}

\author{
Martin Greiter \\ Department of Physics, Stanford University, Stanford, CA 94305, greiter@quantum.stanford.edu
}

(SU-ITP 98/20, cond-mat/9805112, May 10, 1998)

\begin{abstract}
The spinon and holon excitations of two-leg Heisenberg or lightly doped $t-J$ ladders are shown to be bound in pairs by string confinement forces given approximately by the antiferromagnetic exchange energy across the rungs, $F=J_{\perp}\left\langle\mathbf{S}_{i} \mathbf{S}_{j}\right\rangle_{\perp} / b$. These forces originate from the fictitious flux tubes associated with the half-fermi statistics of the excitations. It is conjectured that similar confinement forces, determined by the antiferromagnetic exchange energy across the layers, are responsible for the spin gap and the pairing of charge carriers in $\mathrm{CuO}$ superconductors.
\end{abstract}

PACS numbers: 71.10.-w, 75.10.-b, 74.72.-h, 74.20.-z

1. Two-leg Heisenberg or lightly doped $t-J$ ladders [1] can, according to a recent proposal [2], be described approximately by an RVB type spin liquid. To be precise, one assumes a magnetic tight-binding model on the ladder, with flux $\pi$ per plaquet and hopping magnitudes $\tilde{t}$ along the chains and $\tilde{t}_{\perp}$ across the rungs. One then fills the lower band twice, once with up-spin and once with down-spin electrons. Upon elimination of doubly occupied sites via Gutzwiller projection, one obtains a reasonable approximation [2] to the ground state of the Heisenberg ladder with $J_{\perp} / J=\tilde{t}_{\perp} / \tilde{t}$.

Spinon and holon excitations for this liquid may be created either via Anderson's projection technique [3] or via midgap states 酒. As the topology of the ladder dictates that midgap states can only be created in pairs, the second method automatically yields spinon-spinon bound states (magnons) rather then isolated spinons, which reflects the fact that the spinons or holons are confined in pairs.

In the first part of this letter, I will explain the form and origin of the confinement forces between the spinons and holons of the ladder, and calculate the spinon mass and the bound state resonances based on a heuristic identification of the spin gap of the Heisenberg ladder as the zero point energy of the string oscillator. In the second part, I will postulate similar confinement forces in systems of (weakly) coupled magnetic planes, make some assumptions regarding both the nature of the spin liquid in the planes and the confinement forces due to the interplane coupling, and obtain an estimate for the spin and charge gaps in bilayer $\mathrm{CuO}$ superconductors $(25 \mathrm{meV}$ and $28 \mathrm{meV}$, respectively, for $\mathrm{YBa}_{2} \mathrm{Cu}_{3} \mathrm{O}_{6+x}$ ). I conclude with several comments on high- $T_{c}$ superconductivity.

2. In order to determine the functional form and strength of the confining potential between the spinon or holon excitations of the two-leg Heisenberg ladder, we create two holons at sites $i$ and $j$ via Anderson's projection technique [3],

$$
\left|\psi_{i, j}\right\rangle=c_{i \uparrow} c_{j \downarrow} \mathcal{P}_{\mathrm{G}} c_{i \uparrow}^{\dagger} c_{j \uparrow}\left|\psi_{\mathrm{SD}}\right\rangle
$$

where $\left|\psi_{\mathrm{SD}}\right\rangle$ is the Slater determinant ground state obtained by filling the lower magnetic tight-binding band

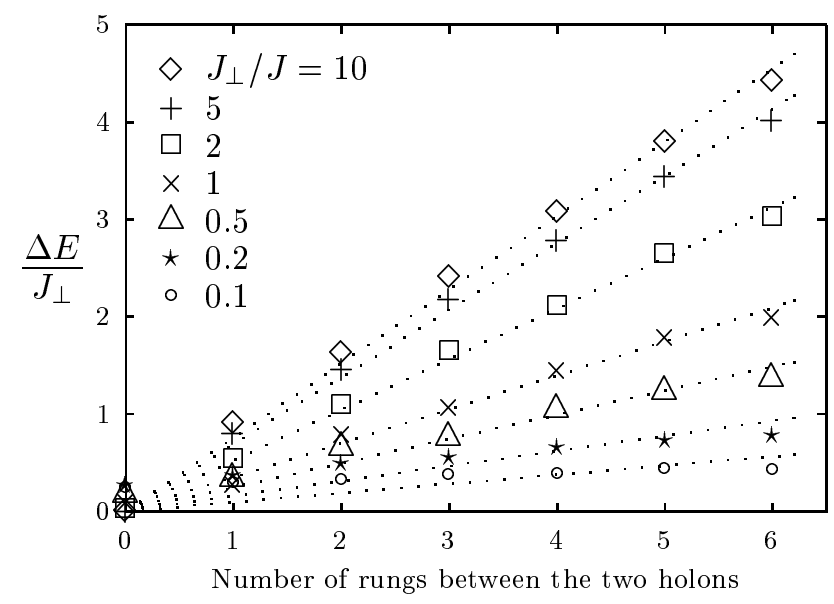

FIG. 1. The confinement energy, defined here as the energy of a pair of holons measured relative to the exact ground state energy of a pair of holes, as a function of the number of rungs between the holons or holes for a $2 \times 8$ ladder with open boundary conditions and $\tilde{t}_{\perp} / \tilde{t}=0.75\left(J_{\perp} / J\right)^{0.5}$ for $J_{\perp} / J \leq 1$ and $\tilde{t}_{\perp} / \tilde{t}=\left(J_{\perp} / J\right)^{0.6}$ for $J_{\perp} / J \geq 2$, i.e. such that the ratios $\left\langle\vec{S}_{i} \vec{S}_{j}\right\rangle_{\perp} /\left\langle\vec{S}_{i} \vec{S}_{j}\right\rangle_{\|}$of the trial wave functions match those of the exact ground states. One holon was localized at the end of the ladder and the other localized at various positions on the same chain, as illustrated in fig. 3a; a finite size correction has been taken into account for $J_{\perp} / J=0.2$ and 0.1 .

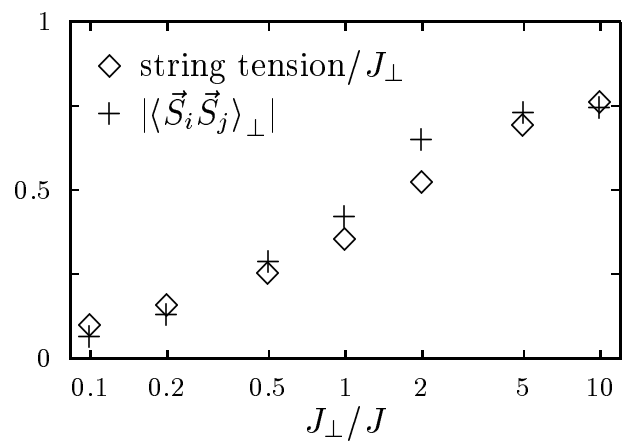

FIG. 2. The string tension in units of $J_{\perp}$ given by the slope of the dotted lines fitted though the data points in fig. 1 in comparison with the spin correlations $\left|\left\langle\vec{S}_{i} \vec{S}_{j}\right\rangle_{\perp}\right|$ across the rungs in the ground state as taken from table I of [2] for different ratios $J_{\perp} / J$. The discrepancy around $J_{\perp} / J=2$ arises from the enhanced correlations $\left|\left\langle\vec{S}_{i} \vec{S}_{j}\right\rangle_{\|}\right|$along the chains in the presence of the holons, which have been neglected in (2). 


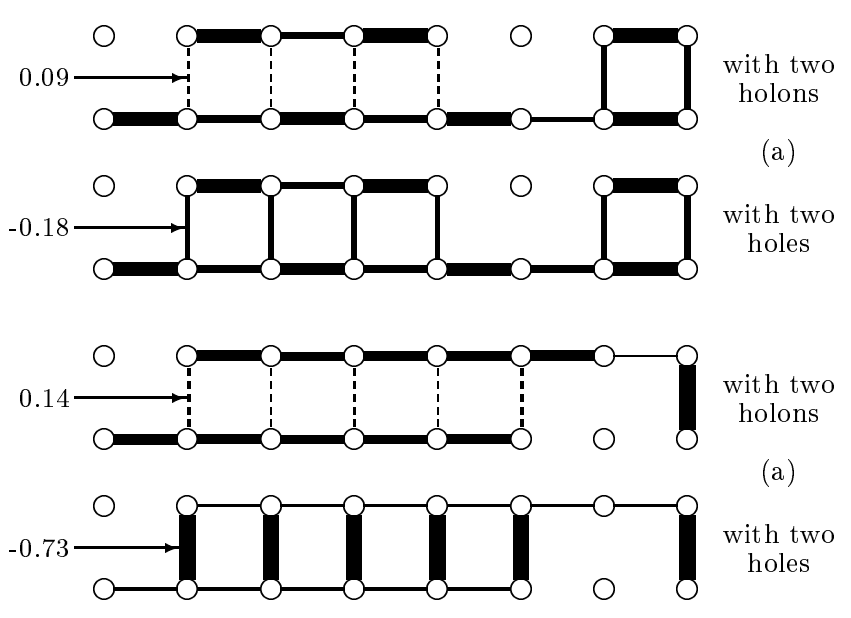

FIG. 3. Spin correlations $\left\langle\vec{S}_{i} \vec{S}_{j}\right\rangle$ between nearest neighbors in the presence of two holons compared with two holes for two representative configurations of a $2 \times 8$ ladder with open boundary conditions and (a) $J_{\perp} / J=0.5$ or (b) $J_{\perp} / J=5$. The conventions are adopted from White and Scalapino [6] the thickness of the lines is proportional to $\left|\left\langle\vec{S}_{i} \vec{S}_{j}\right\rangle\right|$; solid lines indicate antiferromagnetic, dotted lines ferromagnetic correlations. The state containing two holons was obtained from Gutzwiller projected magnetic bands using Anderson's method for constructing spinons; the state containing two holes is just the exact ground state of the Heisenberg ladder with two static vacancies at the positions indicated.

twice, and numerically compare the energy expectation value of this configuration to the energy of the exact ground state for a Heisenberg ladder with two stationary holes at these sites [5]. The results are shown fig. 11 and 2; we find a linear potential

$$
V(x)=F|x| \quad F \approx J_{\perp}\left|\left\langle\mathbf{S}_{i} \mathbf{S}_{j}\right\rangle_{\perp}\right| / b
$$

where $x$ is the distance between the spinons or holons in the direction of the chains, $\left\langle\mathbf{S}_{i} \mathbf{S}_{j}\right\rangle_{\perp}$ the spin correlation across the rungs, and $b$ the bondlength along the chains. The confinement energy is hence approximately equal to the antiferromagnetic exchange energy across the rungs between the spinons; the reason for this emerges from a comparison of the individual spin correlations on each link, as reproduced in fig. 3 for two typical configurations: an invisible string between the holons destroys the antiferromagnetic correlations on all the rungs between them.

The origin of this string is explained in fig. 4: the fictitious flux tube associated with the half-fermi statistics [8] of the spinons or holons [7],9], which manifests itself in an adjustment by $\pi$, 4,2] of the fictitious flux through the adjacent plaquets in the magnetic tight-binding model before Gutzwiller projection, effectively annihilates the hopping terms on the rungs between them.

3 . If we were to know the effective mass of the spinon, we could calculate the resonances of the string oscillator
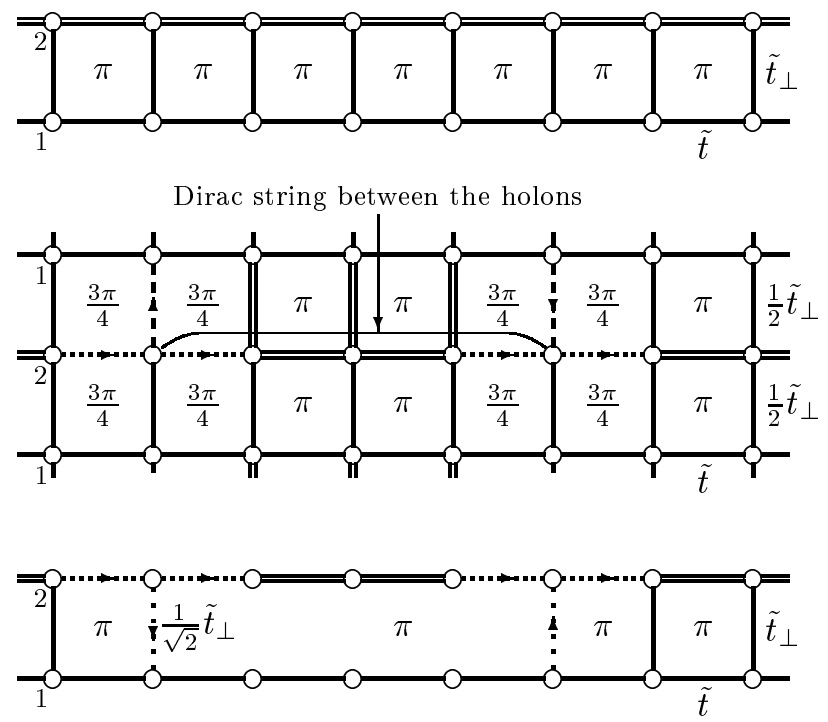

Conventions for hopping phases across links:

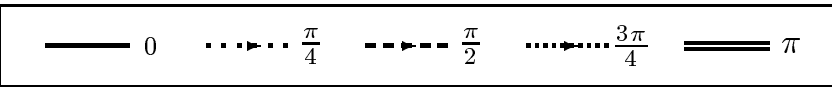

FIG. 4. Magnetic tight-binding configurations before Gutzwiller projection for (a) the ground state of the $t-J$ ladder at half-filling and (b,c) in the presence of two holons at a distance of 4 lattice spacings. The holons in (b) were created following the procedure for the Kalmeyer-Laughlin chiral spin liquid [7] suggested in [2] after mapping the flux ladder into a flux lattice subject to a periodic boundary condition with a periodicity of only two lattice spacings in $y$-direction. The lattice is subsequently reconverted into a ladder (c). Note that the Dirac string annihilates all the hopping terms across the rungs between the holons, while the hopping magnitudes along the chains remain unaffected. 


$$
\left(-\frac{1}{2 m_{\text {red }}} \nabla^{2}+F|\mathbf{r}|\right) \psi_{n}(\mathbf{r})=E_{n} \psi_{n}(\mathbf{r}),
$$

where $m_{\mathrm{red}}=\frac{1}{2} m_{\mathrm{sp}}$ is the reduced mass of a spinon pair, and compare the ground state energy to the spin gap in the Heisenberg ladder, which is just the energy required to create a spinon-spinon bound state; apart from a possible correction due to a chemical potential for spinons which we neglect, these should be equal. In the present case, we use the know value $\Delta \approx J_{\perp} / 2$ for the spin gap [10] to calculate the spinon mass and the spinon-spinon resonances in the weak coupling regime $J_{\perp}<J$. In one dimension, the solutions of (3) are given in terms of the Airy function $\operatorname{Ai}(x)$ [1],

$$
\psi_{n}(x)=\left(\frac{x}{|x|}\right)^{n} \operatorname{Ai}\left(\frac{|x|}{x_{0}}-\lambda_{n}\right), \quad E_{n}=F x_{0} \lambda_{n},
$$

where $x_{0}=1 /\left(2 m_{\mathrm{red}} F\right)^{1 / 3}$ is the characteristic lengthscale of the oscillator, and $\lambda_{n}$ are the extrema or zeros of $\operatorname{Ai}(-x)$ for $n$ even or $n$ odd, respectively, which are listed in table 1 . Equating $E_{0}=\Delta$, we obtain

$$
m_{\mathrm{sp}}=\frac{F^{2} \lambda_{0}^{3}}{E_{0}^{3}}=\frac{8\left|\left\langle\mathbf{S}_{i} \mathbf{S}_{j}\right\rangle_{\perp}\right|^{2} \lambda_{0}^{3}}{J_{\perp} b^{2}}=3.25 \frac{J_{\perp}}{J^{2} b^{2}},
$$

where we have approximated $\left|\left\langle\mathbf{S}_{i} \mathbf{S}_{j}\right\rangle_{\perp}\right| \approx 0.62 J_{\perp} / J$ according to table I of [2] for weakly coupled ladders. The spacing of the eigenvalues $\lambda_{n}$ implies that the internal resonance frequencies of the spinon-spinon bound states or magnons are comparable but slightly higher than the energies required to create a second or third magnon; this proximity and the fact that they presumably decay rapidly into several magnons renders a potential observation of the resonances difficult if not impossible.

It is now easy to estimate the size of the magnon. Using $\operatorname{Ai}\left(|x|-\lambda_{0}\right) \approx \operatorname{Ai}\left(-\lambda_{0}\right) \exp \left(-\frac{1}{3} x^{2}\right)$, we write the ground state

$$
\psi_{0}(x)=\exp \left(-\frac{x^{2}}{2 \xi^{2}}\right) \text { with } \xi=\sqrt{\frac{3}{2}} x_{0}=0.97 \frac{J}{J_{\perp}} b .
$$

This result illustrates why the spin gap in the weak coupling regime can be $\frac{1}{2} J_{\perp}$ while the antiferromagnetic exchange energy across each rung is only of order $\frac{1}{2} J_{\perp}^{2} / J$ : the number of decorrelated rungs is of order $J / J_{\perp}$.

This calculation can also be applied to spinon-holon bound states (holes) in the ladder; the only difference is that the reduced mass in (3) and (4) is replaced by

$$
\frac{1}{m_{\mathrm{red}}}=\frac{1}{m_{\mathrm{sp}}}+\frac{1}{m_{\mathrm{h}}}, \text { where } m_{\mathrm{h}}=\frac{1}{2 t_{\mathrm{eff}} b^{2}}
$$

is the effective mass of the holon; according to table II of [2], $t_{\mathrm{eff}}=\frac{1}{2} E_{t_{\|}}=0.77 t$ for $J_{\perp}=J$ and $t_{\mathrm{eff}}=0.95 t$ in the weak coupling limit $J_{\perp} \ll J$. The values for the energies $E_{n}$ and the size $\xi$ of the bound state are those given in (4) and (6) above multiplied by

\begin{tabular}{c|cc|ccc}
\hline \hline & \multicolumn{2}{|c|}{ 1d string } & \multicolumn{3}{|c}{ 2d string oscillator } \\
$n$ & $\lambda_{2 n}$ & $\lambda_{2 n+1}$ & $\lambda_{n 0}$ & $\lambda_{n 1}$ & $\lambda_{n 2}$ \\
\hline 0 & 1.0188 & 2.3381 & 1.7372 & 2.8721 & 3.8175 \\
1 & 3.2482 & 4.0879 & 3.6702 & 4.4930 & 5.2629 \\
2 & 4.8201 & 5.5206 & 5.1697 & 5.8671 & 6.5415 \\
\hline \hline
\end{tabular}

TABLE I. The lowest (dimensionless) energy eigenvalues for the linear oscillator in one and two dimensions.

$$
\mu=\left(\frac{1}{2}+\frac{m_{\mathrm{sp}}}{2 m_{\mathrm{h}}}\right)^{1 / 3}=\left(\frac{1}{2}+3.25 \frac{t_{\mathrm{eff}} J_{\perp}}{J^{2}}\right)^{1 / 3}
$$

4. I will now turn to the more speculative part of this letter, and explain part of my thinking on $\mathrm{CuO}$ superconductivity. To begin with, I make the following assumptions: 1 . The ground state of the two-dimensional $t-J$ model at the relevant hole dopings is a spin liquid, which supports spinons and holons as elementary excitations [3]. (My understanding [12] of this liquid is that it is a liquid in both the spin degrees of freedom and in the non-relativistic plaquet chiralities [13]; this chirality liquid may be seen as a significant generalization of Laughlin's chiral spin liquid [0], which is a liquid in the spins but effectively aligns the chiralities and thereby violates the discrete symmetries $\mathrm{P}$ and $\mathrm{T}$. These symmetries are preserved in my construction. The spinon and holon excitations supported by the chirality liquid carry a chirality quantum number, which can be + or - ; this number determines the sign of the winding phases associated with their half-fermi statistics.) 2. The spinon and holon masses in a system of coupled $t-J$ planes are comparable to their values in a system of coupled chains estimated above. 3. The analysis above, including all the implicit assumptions made, is correct.

The main difference between fictitious flux confinement in a system of coupled planes as compared to coupled chains is that the one-dimensional array of decorrelated rungs is replaced by a puddle of decorrelated interplane links. To see this, imagine several ladders (which are not necessarily straight) embedded into a system of coupled planes such that the rungs align with interplane links, and connect two spinon sites $i$ and $j$ along various paths in the planes with these ladders; the fictitious flux connecting the spinons will then destroy the correlations across all the rungs on each ladder.

The simplest estimate for the strength of the confining force is to assume a circular droplet of decorrelated links between the spinons, with a diameter given by the spinon-spinon distance $r$. (This is presumably not a valid approximation for large spinon separations, but may be reasonable for the ground state of the oscillator.) It yields a harmonic potential

$$
V(r)=\frac{\pi r^{2}}{4 b^{2}} J_{\perp}\left|\left\langle\mathbf{S}_{i} \mathbf{S}_{j}\right\rangle_{\perp}\right| \equiv \frac{1}{2} D r^{2} .
$$

The ground state energy of the spinon-spinon bound 


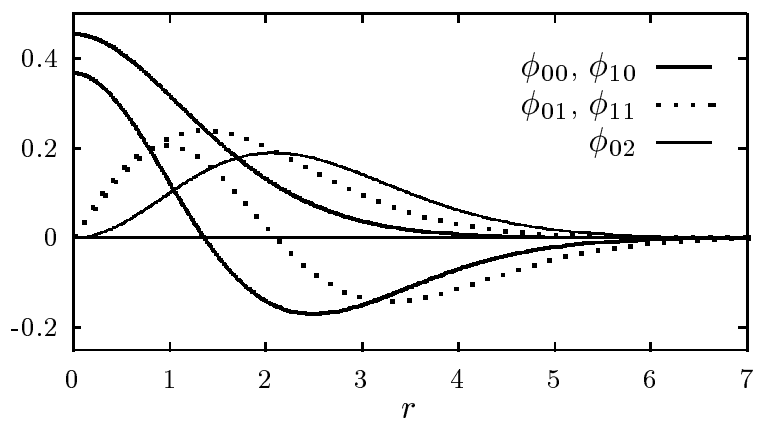

FIG. 5. Radial wave functions for the lowest energy eigenstates of the linear oscillator in two dimensions.

state is hence given by

$$
E_{0}=\sqrt{\frac{D}{m_{\text {red }}}}=\sqrt{\frac{\pi}{3.25}\left|\left\langle\mathbf{S}_{i} \mathbf{S}_{j}\right\rangle_{\perp}\right| J^{2}}=0.77 \sqrt{J_{\perp} J},
$$

where we have used (5) and $\left|\left\langle\mathbf{S}_{i} \mathbf{S}_{j}\right\rangle_{\perp}\right| \approx 0.62 J_{\perp} / J$. This estimate for the spin gap [14] in $\mathrm{YBa}_{2} \mathrm{Cu}_{3} \mathrm{O}_{6+x}$ is directly related to the optical magnon gap in the ordered antiferromagnet $\mathrm{YBa}_{2} \mathrm{Cu}_{3} \mathrm{O}_{6.2}$, which has been measured by inelastic neutron scattering 15 :

$$
E_{\text {opt. }}=2 \sqrt{J_{\perp} J} \approx 70 \mathrm{meV},
$$

which yields a spin gap of $27 \mathrm{meV}$. The charge gap is just the gap to create a spinon-holon bound state: substituting $J=120 \mathrm{meV}, J_{\perp}=10 \mathrm{meV} t=500 \mathrm{meV}$, and $t_{\text {eff }}=0.9 t$ for $\mathrm{YBa}_{2} \mathrm{Cu}_{3} \mathrm{O}_{6+x}$ into (8), we obtain $31 \mathrm{meV}$.

The microscopic details of the chirality liquid [12] yield a linear potential as an estimate for the confining force,

$$
F_{2 \mathrm{~d}}=\frac{1}{b} \sqrt{\frac{E_{00} J_{\perp}\left|\left\langle\mathbf{S}_{i} \mathbf{S}_{j}\right\rangle_{\perp}\right|}{2}}
$$

where $E_{00}$ is the spin gap given by the ground state energy of the string oscillator (3) with string tension $F_{2 \mathrm{~d}}$ in two dimensions. This oscillator has to be solved numerically; the solutions are

$$
\psi_{n \ell}(r, \varphi)=e^{ \pm i \ell \varphi} \phi_{n \ell}\left(\frac{r}{r_{0}}\right), E_{n \ell}=F_{2 \mathrm{~d}} r_{0} \lambda_{n \ell},
$$

with $r_{0}=1 /\left(2 m_{\text {red }} F_{2 \mathrm{~d}}\right)^{1 / 3}, \lambda_{n \ell}$ as listed in table [1, and $\phi_{n \ell}(r)$ as plotted in fig. 5 .

Solving (12) and (13) for $E_{00}$ yields

$$
E_{00}=\sqrt{\frac{J_{\perp}\left|\left\langle\mathbf{S}_{i} \mathbf{S}_{j}\right\rangle_{\perp}\right| \lambda_{00}^{3}}{4 m_{\mathrm{red}}}}=\sqrt{\frac{1}{2} J_{\perp} J}
$$

where we have used (5) and $\left|\left\langle\mathbf{S}_{i} \mathbf{S}_{j}\right\rangle_{\perp}\right| \approx 0.62 J_{\perp} / J$ once more. This yields a spin and charge gap of $25 \mathrm{meV}$ and $28 \mathrm{meV}$ for $\mathrm{YBa}_{2} \mathrm{Cu}_{3} \mathrm{O}_{6+x}$.

It should of course be born in mind that these numbers are only rough estimates; many important details, including the $d$-wave symmetry of the superconducting order parameter, have not been taken into account here.

5. I wish to conclude with a few general observations relating the circle of ideas drawn above to high- $T_{\mathrm{c}}$ superconductivity.

(a) The fictitious flux confinement forces are not responsible for the pairing of the charge carriers in a conventional sense, as the charge superfluid forms an integral part of the spin liquid within the planes; in particular, the interplane correlations are not destroyed by the presence of charge carriers in the superconducting state at $T=0$. The charge gap estimated above, however, corresponds roughly [16] to the gap required to create an "unpaired" hole, which does not participate in the superfluid. The confinement forces are thus responsible for the robustness of the pairing, including its persistence at finite temperatures, but not for the pairing itself 17 .

(b) The strength of the magnetic pairing force is determined by the the magnetic energy stored in the structure between the layers, or more precisely by the amount this energy is raised by the fictitious flux, which is only in multilayer materials directly related to the coupling between the layers. The mechanism hence allows for strong pairing forces in single layer materials; we expect those, however, to be crucially dependent on the magnetic properties of the structure between the planes.

(c) My thinking on the spin gap phase coincides with that of Emery and Kivelson 18 .

(d) The superconductivity in $\mathrm{C}_{60}$ is, according to my thinking, due to Dirac quantization of the fictitious flux through the surface of each molecule [19,20,12].

I am deeply grateful to S. Chakravarty, A. Kapitulnik, S. Kivelson, but especially R.B. Laughlin for many illuminating discussions. This work was supported through NSF grant No. DMR-95-21888. Additional support was provided by the NSF MERSEC Program through the Center for Materials Research at Stanford University.

[1] For a review see E. Dagotto and T.M. Rice, Science 271, 618 (1996).

[2] M. Greiter, The two-leg $t-J$ ladder: a spin liquid generated by Gutzwiller projection of magnetic bands, condmat/9804002.

[3] P.W. Anderson in Frontiers and Borderlines in Many Particle Physics, edited by J.R. Schrieffer et al. (NorthHolland, 1988); Science 235, 1196 (1987).

[4] D.S. Rokhsar, Phys. Rev. Lett. 65, 1506 (1990).

[5] Strictly speaking, the exact ground state contains a small admixture of a state with two holons, which we have neglected here as it tends to zero as the coupling $J_{\perp} / J$ gets stronger or as $i$ and $j$ get separated further.

[6] S.R. White and D.J. Scalapino, Phys. Rev. B 55, 6504 (1997).

[7] V. Kalmeyer and R.B. Laughlin, Phys. Rev. B 39, 11897 (1989); R.B. Laughlin and Z. Zou, ibid. 41, 664 (1990).

[8] For a review see F. Wilczek, Fractional statistics and anyon superconductivity (World Scientific, 1990). 
[9] F.D.M. Haldane, Phys. Rev. Lett. 67, 937 (1991).

[10] T. Barnes, E. Dagotto, J. Riera, and E.S. Swanson, Phys. Rev. B 47, 3196 (1993); D.G. Shelton, A.A. Nersesyan, and A.M. Tsvelik, ibid. 53, 8521 (1996).

[11] See for example M. Abramowitz and I.A. Stegun (eds.), Handbook of Mathematical Functions (Dover, 1964).

[12] M. Greiter, SU-ITP 98/37 and 98/38, in preparation.

[13] X.G. Wen, F. Wilczek, and A. Zee, Phys. Rev. B 39, 11413 (1989)

[14] For a review see N.P. Ong, Science 273, 321 (1996).

[15] D. Reznik et al., Phys. Rev. B 53, R14741 (1996); S.M. Hayden et al., ibid. 54, R6905 (1996).

[16] The superconducting gap $\Delta$ is slightly larger than the charge gap estimated as kinetic energy is gained through interlayer tunneling; see S. Chakravarty, condmat/9801025, and references therein.

[17] The superfluid itself may belong to the general class of topological superconductors studied by P.B. Wiegmann, Prog. of Theo. Phys. Suppl. 107243 (1992).

[18] S. Doniach and M. Inui, Phys. Rev. B 41, 6668 (1990); V.J. Emery and S.A. Kivelson, Nature 374, 434 (1995).

[19] A.F. Hebard et al., Nature 350 (1991); K. Holczer et al., Science 252 (1991).

[20] S. Chakravarty, M. Gelfand, and S. Kivelson, Science 254 (970). 